\title{
25 Research Square \\ Curative Effect of Oblique Lateral Interbody Fusion in the Treatment of Lumbar Tuberculosis
}

\section{Wenhui Zhang}

Gansu Provincial Hospital

\section{Weiqiang Sun}

Traditional Chinese Medicine hospital of Zhang country

\section{Yuanyuan Zhang}

Gansu University of Traditional Chinese Medicine

\section{Fengguang Yang}

Gansu Provincial Hospital

\section{Yuping Yang}

Gansu Provincial Hospital

Mingdong lu

Gansu Provincial Hospital

\section{Shifang Guo}

Gansu Provincial Hospital

\section{Zongru He}

Gansu Provincial Hospital

Jie Liu (D787159913@qq.com)

Gansu Provincial Hospital

\section{Research article}

Keywords: Lateral oblique interbody fusion, Lumbar tuberculosis, Minimally invasive, Surgical approach

Posted Date: October 5th, 2021

DOI: https://doi.org/10.21203/rs.3.rs-880038/v1

License: (c) (i) This work is licensed under a Creative Commons Attribution 4.0 International License.

Read Full License 


\section{Abstract}

Objective: This research aimed to investigate the curative effect of oblique lateral interbody fusion (OLIF) in the treatment of lumbar tuberculosis.

Methods: A total of 32 patients, including 18 males and 14 females (29-58 years old, with the mean age of 47) who were admitted to our hospital from October 2016 to June 2020 and treated with oblique lateral interbody fusion combined with vertebral screw internal fixation, were retrospectively collected. The segmental distribution of lesions was $L_{2}-L_{3} 16$ cases, $L_{3}-L_{4} 11$ cases and $L_{4}-L_{5} 5$ cases. At first, preoperative antituberculous drug treatment was standardized for 2-4 weeks. Furthermore, intraoperative blood loss, operation time, postoperative complications, ESR, CRP, VAS, JOA and ASIA scores were recorded before and after surgery and at the last follow-up. In addition, antituberculous drug therapy was regulated continuously after surgery.

Results: All patients were followed up for 12-17 months, with an average of 14 months. $\otimes$ The average operation time was $(132.5 \pm 21.4) \mathrm{min}$, and the intraoperative blood loss was $(227.3 \pm 43.1) \mathrm{ml}$. $\nabla$ Preoperative ESR was $(74.56 \pm 15.43) \mathrm{mmol} / \mathrm{h}$, and CRP was $(43.21 \pm 19.37) \mathrm{mg} / \mathrm{L}$. Seven days after surgery, ESR was $(42.37 \pm 19.26) \mathrm{mmol} / \mathrm{h}$, and CRP was $(22.51 \pm 18.42) \mathrm{mg} / \mathrm{L}$. In the last follow-up, ESR was $(9.25 \pm 4.13) \mathrm{mmol} / \mathrm{h}$, and CRP was $(8.17 \pm 3.21) \mathrm{mg} / \mathrm{L}$. They have statistically significant differences before surgery, seven days after the surgery and in the last follow-up $(P<0.05)$. $\otimes$ The average VAS score before surgery was $(7.21 \pm 1.40)$ points, the average JOA score was $(18.24 \pm 4.13)$ points, the average VAS score seven days after surgery was $(2.23 \pm 1.32)$ points, the average JOA score was $(24.72 \pm 3.19)$ points, and the average VAS score in the last follow-up was $(1.63 \pm 0.54)$ points. JOA score was $(27.72 \pm 2.61)$. They have statistically significant differences before surgery, seven days after the surgery and in the last follow-up $(P<0.05) \otimes$ Six patients had neurologic symptoms with ASIA grade $D$ before surgery, 2 patients recovered to Grade $E$ seven days after surgery, and the remaining 4 patients also recovered to grade $E$ in the last follow-up. Besides, 26 patients were graded as Grade E before surgery, seven days after surgery and in the last follow-up. $\otimes$ After surgery, 1 patient suffered psoas major muscle damage, which was manifested as reduced hip flexion strength of the lower extremity, and returned to normal three weeks later. Apart from that, none of the patients had complications such as abdominal organ injury and poor wound healing.

Conclusion: OLIF is a new minimally invasive method for treating lumbar tuberculosis, with short operation time, less blood loss, convenient operation, thorough removal of lesions, and good long-term follow-up effect. It is worthy of promotion and application.

\section{Introduction}

Tuberculosis (TB) is one of the oldest diseases affecting human health. With the development of medicine and the enhancement of people's concept of medical treatment, the incidence of tuberculosis is gradually decreasing, whereas drug-resistant TB is rising ${ }^{[1,2]}$. Among extra-pulmonary tuberculosis, bone 
and joint tuberculosis accounts for about 1\%-3\% [3], and spinal tuberculosis occupies the largest proportion ${ }^{[4]}$. Among the spinal tuberculosis, thoracic-lumbar tuberculosis is the most common, accounting for about $48 \%$ of the thoracic vertebra tuberculosis and about $42 \%$ of the lumbar vertebra tuberculosis respectively, while cervical tuberculosis and sacrococcygeal tuberculosis are rare ${ }^{[5]}$. For some patients with slight clinical lesions, good effect can be obtained through classical quadruple drug treatment ${ }^{[6-8]}$. However, part of the spinal tuberculosis with abscess, spinal nerve compression or damage after spinal stability caused the collapse of the anterior column protrusion deformity, and so on, all requiring surgical intervention treatment. At present, it is also the emphasis and difficulty in the spinal tuberculosis treatment ${ }^{[9]}$.

Common surgical approaches for spinal tuberculosis mainly include anterior, posterior, combined anterior and posterior approaches, etc. ${ }^{[10]}$. Nonetheless, different approaches have their own advantages and disadvantages ${ }^{[11-12]}$. Thus, their clinical use is also limited accordingly. In 2012, a new minimally invasive technique was first reported by French doctor Silvestre et al. Oblique Interbody Fusion, which enters the extraperitoneal space through the external Oblique, internal Oblique and transverse abdominis to reach the lateral side of the lumbar spine, has some advantages such as greatly shortening the operation time, and reducing intraoperative bleeding and trauma. Therefore, it has been widely applied in

the treatment of lumbar degenerative changes and has also achieved satisfactory results ${ }^{[13-15]}$. However, the effect of this operation in lumbar tuberculosis is not reported.

From October 2016 to June 2020, our hospital had adopted OLIF to treat 32 cases of patients with lumbar tuberculosis, and achieved good efficacy. The report is as follows:

\section{Data And Methods}

\subsection{General Information}

A total of 32 patients, including 18 males and 14 females (29-58 years old, with an average age of 47) who suffered spinal tuberculosis and received OLIF operation in our hospital from October 2016 to June 2020, were selected. The segmental distribution of lesions was L 2-L316, L3-L411, and L4-L55. Meanwhile, none of the patients had significant kyphosis. The mean preoperative erythrocyte sedimentation rate $(E S R)$ was $(74.56 \pm 15.43) \mathrm{mmol} / \mathrm{h}$, and the preoperative mean value of hypersensitive C-reactive protein (CRP) was $(43.21 \pm 19.37) \mathrm{mg} / \mathrm{L}$. Beyond that, the mean Visual Analogue Scale (VAS) score and Japanese Orthopaedic Association (JOA) score were $(7.21 \pm 1.40)$ and $(18.24 \pm 4.13)$ respectively. All the patients presented with different degree of low back pain and discomfort or accompanied by fever, night sweats and fatigue. In addition, 6 patients had lower extremity's sensory and motor functional limitation. The American Spinal Injury Association (ASIA) grading results were D.

\subsection{Inclusion Criteria}


$\nabla$ It is based on the patient's symptoms, signs, laboratory examination, physical examination, and other factors consistent with the diagnosis of spinal tuberculosis. $\nabla$ Drug treatment failed after 2-4 weeks. $\nabla$ The lesion is relatively limited: being confined to a single intervertebral space or adjacent vertebral body. $\otimes$ Patients have clear indications (e.g.obvious damage to the vertebral body, and severe collapse of the anterior column, dead bone or cavity in the vertebral lesions). $\nabla$ According to preoperative evaluation, patients are generally in a good condition and can tolerate the operation. $\otimes$ Patients and their families agree to the operation.

\subsection{Exclusion Criteria}

$\nabla$ Patients have serious lesion invasion of spinal canal and severe spinal cord compression. $\otimes$ Preoperative assessment of general intolerability of surgery. खPostoperative pathological examination of non-tuberculosis. $\otimes$ Patients have other spinal diseases. $\otimes$ Patients refuse surgery and have incomplete case data. $\otimes$ Patients' lesions are located at $L_{5}-S_{1}$.

\subsection{Treatment methods}

\subsubsection{Preoperative treatment}

Prior to surgery, all the patients were treated with antituberculous drugs for 2-4 weeks. Classic four-drug combination - Isoniazid (H), Rifampin (R), Ethambutol (E) and Pyrazinamide (Z) - was used. Due to the hepatotoxicity of Rifampin, hepatophane therapy was taken simultaneously. The liver function of the patients was tested regularly. After standard antituberculous drug treatment, surgical treatment should be arranged timely if the patient's systemic symptoms were improved, ESR and CRP were significantly decreased compared with those before treatment, and there were no fever, anemia and other symptoms. If psoas major abscess is present before surgery, it is essential to perform puncture and drainage.

\subsubsection{Surgical Treatment}

After the patient was anesthetized, the right decubitus position was taken and the towel was disinfected. First, part of the iliac bone was taken from the anterior superior iliac spine for backup, and preoperative planning of the surgical incision site was conducted. The next is to cut the skin, subcutaneous tissue, the external oblique abdominal muscle, internal oblique muscle, the transverse abdominal muscle, and muscle fiber line direction, separate muscle tissue into the peritoneal clearance, show lesions of vertebral body parts, pull open area with retractor, remove tissue, destroy the intervertebral disc, pus, and dead bone with hydrogen peroxide solution and iodine volts. Furthermore, the previously removed iliac bone was rested to an appropriate length and placed between the vertebrae. In addition, vertebral nails were inserted into the upper and lower vertebrae in the focus area. Apart from that, the vertebral nail position and muscle direction were examined by fluoroscopy.

\subsubsection{Postoperative treatment}


The intraoperative lesion tissues were sent for pathological examination, laboratory microbial culture, and bacterium tuberculosis DNA examination. At the same time, standard antituberculous drugs were used continuously for treatment. The waist brace was protected and fixed, and the patient rested in bed for one month. One month later, the patient wore the chest and waist brace to move, but spinal weight bearing and bending and rotating activities should be avoided. The liver and kidney functions, CRP and ESR were tested regularly, and the medication was adjusted timely according to the test results. Besides, other symptomatic and supportive treatment could be conducted.

\subsection{Evaluation Indexes}

(1) Operation time ( $\mathrm{min}),(2)$ intraoperative blood loss ( $\mathrm{ml}),(3)$ VAS score before and after surgery, (4) JOA score before and after surgery, (5) CRP and ESR before and after surgery, (6) postoperative neurological recovery (ASIA grade), (7) complications.

\subsection{Statistical Analysis}

SPSS 21 statistical software was applied, the data were expressed as percentage and rate, and $\chi 2$ test was used. The measurement data were expressed by (Mean $\pm S D)$ and $t$ test was performed. Additionally, rank sum test was used for grade data, and the test level was 0.05 . Moreover, $P<0.05$ indicated that the difference was statistically significant.

\section{The Results}

\subsection{Surgical Results}

Postoperative pathological examination of all patients in this study showed caseous tissue, suggesting lumbar tuberculosis infection. The average operation time of the patients was (132.5 \pm 21.4$) \mathrm{min}$, and the intraoperative blood loss was $(227.3 \pm 43.1) \mathrm{ml}$ (see the results in Table 1).

\subsection{ESR and CRP}

Preoperative ESR was $(74.56 \pm 15.43) \mathrm{mmol} / \mathrm{h}$ and CRP was $(43.21 \pm 19.37) \mathrm{mg} / \mathrm{L}$. Seven days after surgery, ESR was $(42.37 \pm 19.26) \mathrm{mmol} / \mathrm{h}$, and CRP was $(22.51 \pm 18.42) \mathrm{mg} / \mathrm{L}$. In the last follow-up, ESR was $(9.25 \pm 4.13) \mathrm{mmol} / \mathrm{h}$, and CRP was $(8.17 \pm 3.21) \mathrm{mg} / \mathrm{L}$. They have statistically significant difference before surgery, seven days after surgery and in the last follow-up. In addition, $\mathrm{P}<0.05$ (see the results in Table 2)

\subsection{VAS score and JOA score}

The average VAS score before surgery was $(7.21 \pm 1.40)$ points, the average JOA score was $(18.24 \pm 4.13)$ points, the average VAS score seven days after surgery was $(2.23 \pm 1.32)$ points, the average JOA score was $(24.72 \pm 3.19)$ points, and the average VAS score in the last follow-up was $(1.63 \pm 0.54)$ points. JOA 
score was (27.72 \pm 2.61$)$. They have statistically significant difference before surgery, seven days after surgery, and in the last follow-up. Moreover, $\mathrm{P}<0.05$ (see the results in Table 3).

\subsection{ASIA grade}

Among a total of 32 patients, 6 patients had neurologic symptoms with ASIA grade D before surgery, and 2 patients recovered to Grade $E$ seven days after surgery. The remaining 4 patients also recovered to Grade $\mathrm{E}$ in the last follow-up. Meanwhile, 26 patients were graded as Grade E before surgery, seven days after surgery and in the last follow-up (see the statistical results in Table 4).

\subsection{Complications}

One patient suffered psoas major muscle damage after surgery, which was manifested as reduced hip flexion strength of the lower extremity, and returned to normal three weeks later. None of the patients had complications, such as abdominal organ injury and poor wound healing.

Table 1: Statistical table of operation time and intraoperative blood loss

\begin{tabular}{lll} 
& Range & Mean \pm SD \\
\hline Duration of operation $(\mathrm{min})$ & $121-174$ & $132.5 \pm 21.4$ \\
\hline Intraoperative blood loss $(\mathrm{ml})$ & $182-243$ & $227.3 \pm 43.1$
\end{tabular}

Table 2: Statistical results of ESR and CRP before and after surgery

\begin{tabular}{|c|c|c|}
\hline & ESR『Mean \pm SD $\rrbracket$ & CRP $₫$ Mean $\pm S D$ \\
\hline preoperative & $74.56 \pm 15.43$ & $43.21 \pm 19.37$ \\
\hline 7 days after surgery & $42.37 \pm 19.26$ & $22.51 \pm 18.42$ \\
\hline The last follow-up & $9.25 \pm 4.13$ & $8.17 \pm 3.21$ \\
\hline$P$ value & $<0.05$ & $<0.05$ \\
\hline
\end{tabular}

Table 3: Statistical results of VAS score and JOA score before and after surgery

\begin{tabular}{|c|c|c|}
\hline & 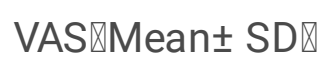 & JOA『Mean \pm SD $\rrbracket$ \\
\hline preoperative & $7.21 \pm 1.40$ & $18.24 \pm 4.13$ \\
\hline 7 days after surgery & $2.53 \pm 1.32$ & $24.72 \pm 3.19$ \\
\hline The last follow-up & $1.63 \pm 0.54$ & $27.72 \pm 2.61$ \\
\hline$P$ value & $<0.05$ & $<0.05$ \\
\hline
\end{tabular}

Table 4: ASIA grade results of patients before and after surgery 


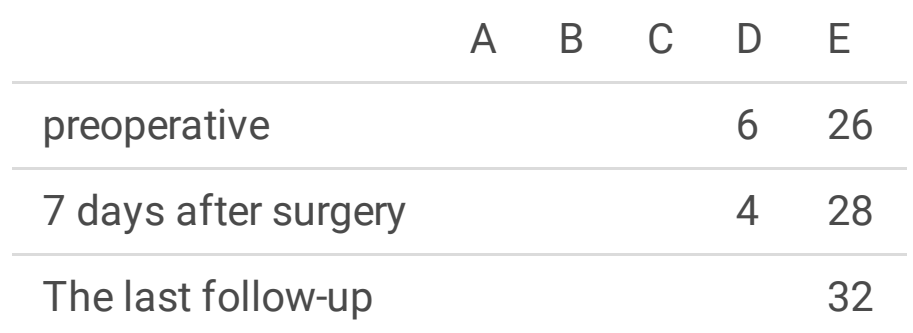

\section{Discussions}

\subsection{Comparison of OLIF and anterior approach}

At present, the common surgical approaches for lumbar tuberculosis mainly include anterior, posterior, combined anterior and posterior approaches, etc. ${ }^{[10] . ~ I n ~ f a c t, ~ m o s t ~ t u b e r c u l o s i s ~ f o c i ~ a r e ~ l o c a t e d ~ i n ~ t h e ~}$ vertebral body and few lesions are located in the adjunct part. Thus, the anterior surgical approach has become the most commonly used surgical approach, which has the advantages such as broad surgical field, direct exposure of the diseased vertebral body during surgery, complete removal of lesions under direct vision, and avoidance of intraoperative tuberculosis bacilli into the posterior column. In this study, the oblique lateral approach was adopted to enter the extraperitoneal space through the external abdominal oblique, internal abdominal oblique and transverse abdominis, and reach the lateral side of the lumbar spine. It can also reach the vertebral body, completely expose the lesion site, and clear the lesion under direct vision, which has the same advantages as the anterior approach.

However, during anterior surgery, doctors need to go through the abdominal cavity and pull the abdominal organs to expose the focus vertebral body. Thus, it has the disadvantages of high risk of injury to the viscera as well as great vessels and more postoperative complications ${ }^{[11-15]}$. According to some scholars, the anterior surgery has a high incidence of false joint, poor correction of deformity and maintenance effect. Meanwhile, long segment screw plate fixation has the risk of screw or titanium plate displacement, fracture and other complications, which is prone to ureter injury, paralytic intestinal obstruction, abdominal organ injury, and other problems ${ }^{[16,17,18]}$. Although some mild organ injuries can be improved after active treatment, they will inevitably cause iatrogenic injuries to the health of patients. If the abdominal aorta is damaged, the life of patients may be endangered in severe cases. Nonetheless, in this study, the oblique lateral approach was adopted to separate the abdominal organs from the operation area by the posterior peritoneum, and the abdominal organs were not directly contacted, so that it not only avoided the risk of injury to the abdominal organs and great vessels, but also made up for the shortcomings of the anterior approach. Among the 13 patients studied, no abdominal organs or great vessels were injured.

\subsection{Comparison of OLIF and posterior approach}

Clinically, with the application of pedicle screw in spinal surgery, posterior surgery has gradually become the main surgical method for treating thoracic tuberculosis. Posterior pedicle screw internal fixation not only enhances postoperative spinal stability, but also significantly corrects the kyphosis of the vertebral 
body caused by tuberculosis ${ }^{[19,20]}$. However, with the application of this surgical method in clinical practice, the disadvantages of posterior surgery have gradually been exposed. To be specific, the anterior column of the spine and anterior vertebral lesions can not completely be removed through simple posterior surgery, which is easy to induce postoperative recurrence. At the same time, there are great disputes on sufficient bone graft support and other issues. In this study, iliac bone was taken from the body, and vertebral lesions and intervertebral discs were removed and placed between two vertebral bodies to support the anterior mid-column of the spine, which also has the advantages of posterior approach surgery in correcting kyphosis of the vertebral body and increasing the stability of the spine. Besides, postoperative review of all cases in this study indicated that the height of the vertebral body was well reconstructed and the stability was good. In comparison to the posterior approach alone, our surgical approach can allow sufficient intervertebral bone grafting, promote postoperative vertebral fusion, increase spinal stability, and overcome the shortcomings of the posterior approach alone. In addition, this method is conductive to completely removing the lesion under direct vision, curetting the dead bone around the lesion, and fully rinsing the lesion area, which can greatly reduce the postoperative recurrence rate, while these advantages are not available in the posterior approach alone.

\subsection{Comparison of OLIF and anterior and posterior combined approaches}

Since the anterior and posterior surgeries have their respective advantages and disadvantages, part of the physicians combine anterior thoracic tuberculosis before and after the treatment. It not only has the advantage of anterior surgery in thoroughly removing lesions and rebuilding the height, but also has the advantage of posterior pedicle screw fixation and better correction of the protruding after spinal deformity ${ }^{[21,22]}$ However, the combined anterior and posterior approach tends to have long operation time, large trauma, more bleeding, high surgical risk, high cost, high technical requirements, and more

postoperative complications, which to some extent limits the application of this surgical method ${ }^{[23,24]}$. According to the previous analysis, the surgical method in this study, namely the oblique lateral approach combined with intervertebral bone graft internal fixation, not only has the advantages of both anterior and posterior approaches, but also has the advantages of simple surgical approach and convenient operation. Therefore, there are not many risks and complications caused by the combined anterior and posterior approaches.

\subsection{Comparison between OLIF and minimally invasive surgery}

In recent years, with the gradual development of minimally invasive concept, clinicians have begun to explore the use of minimally invasive treatment of lumbar spinal tuberculosis technology (e.g. intervertebral foramen lens technology and laparoscopy), in order to achieve the same therapeutic effect as the open surgery and reduce trauma. At the same time, part of the research report of minimally invasive surgery has obtained a good effect ${ }^{[25,26]}$. However, the minimally invasive technique has not been widely applied in clinical practice due to its great difficulty in operation, steep learning curve, as well as certain deficiencies in the complete removal of lesions and the positioning of pedicle screws. By contrast, our study has multiple advantages, including simple operation, easy operation, small trauma, 
and minimally invasive operation. In addition, it is conductive to completely clearing the lesions under direct vision, accurately locating the vertebral nail placement point, and performing vertebral nail internal fixation, thus greatly making up for the deficiency of minimally invasive surgery.

\subsection{Comparison of other indicators}

Q Operative time and intraoperative blood loss: Wu et al. ${ }^{[27]}$ performed anterior surgery, in which the operative time was $(218.5 \pm 11.5) \mathrm{min}$ and intraoperative blood loss was $(663.8 \pm 82.2) \mathrm{ml}$. Apart from that, Zhou et al. ${ }^{[28]}$ performed posterior surgery, with an average operative time of $(160.4 \pm 20.6)$ min and intraoperative blood loss of $(760.7 \pm 146.2) \mathrm{ml}$. Indeed, the average operation time was $(231.5 \pm 27.4) \mathrm{min}$, and the intraoperative blood loss was $(1023.8 \pm 197.9) \mathrm{ml}$. By contrast, the average operation time in our study was $(132.5 \pm 21.4) \mathrm{min}$, and the intraoperative blood loss was $(227.3 \pm 43.1) \mathrm{ml}$. It is obvious that the operative method in this study greatly shortened the operative time and decreased the intraoperative blood loss, no matter which surgical approach was used. $\triangle E S R$ and CRP: Many studies have reported that ESR and CRP after surgery are significantly lower than those before surgery regardless of anterior, posterior or combined anterior and posterior approaches ${ }^{[27,28]}$. In this study, ESR and CRP after surgery were also significantly lower than those before surgery, and there was no significant difference compared with the traditional three surgical approaches. $\triangle$ VAS score and JOA score: As shown by studies, the three traditional surgical approaches can achieve satisfactory results in postoperative VAS score and JOA score ${ }^{[16,18]}$. In this study, the VAS scores and JOA scores of patients before and after surgery had significantly statistical difference, and satisfactory results were also achieved.

\subsection{Supplementary Remarks}

(1) When the lesion was located at L1-L2, if the 12th rib was obstructed during the operation, the leading edge of the 12th rib should be partially excised. As there were no cases in this study with lesions located between L1-L2, partial resection of the leading edge of the 12th rib was not performed. (2) When the lesion was located in the L5 and S1, there was iliac blood vessel damage risk during operation. In this study, we did less operation on lumbar spinal tuberculosis patients, the operation experience was not enough and the operation was too risky. Thus, we temporarily excluded such cases as the standard.

Limitations of this study: The sample size of this study is small. Therefore, a larger sample size and longer follow-up results are needed in the future to further confirm the efficacy of this procedure in the treatment of lumbar tuberculosis.

\section{Conclusion}

In summary, OLIF combined with vertebral screw internal fixation is a new minimally invasive surgical method for the treatment of lumbar tuberculosis, especially in the middle part of the lumbar spine. Its advantages include short operation time, less blood loss, convenient operation, thorough removal of lesions, good follow-up effect, safety and effectiveness. In comparison to the traditional surgical methods, this surgical method not only has many advantages of traditional surgical methods, but also 
can make up for the shortcomings of traditional surgical methods. With good early clinical efficacy for lumbar tuberculosis, it is worthy of clinical promotion and use.

\section{Abbreviations}

Oblique Lateral Interbody Fusion OLIF

Tuberculosis TB

Erythrocyte Sedimentation Rate ESR

C-reactive protein $\quad$ CRP

Visual Analogue Scale VAS

Japanese Orthopaedic Association JOA

American Spinal Injury Association ASIA

Isoniazid $\quad \mathrm{H}$

Rifampin $\quad \mathrm{R}$

Ethambutol $\quad E$

Pyrazinamide $\quad$ Z

\section{Declarations}

Ethics approval and consent to participate consent for publication: Our study was approved by an ethical review board, and all medical records were collected and published with the informed consent of the patients. All authors are informed and agree to publish this article.

Competing interests: The authors have no conflicts of interest to declare.

Funding: This study was funded by the Gansu Provincial Youth Science and Technology Fund (Grant no. 20JR10RA414), Youth Fund of Gansu Provincial Hospital (Grant no. 20GSSY4-30)

Author contributions: Conceived and designed: Wenhui Zhang, Weiqiang Sun, Zongru He, Jie Liu Performed this study: Wenhui Zhang, Weiqiang Sun. Analyzed the date: Wenhui Zhang, Weiqiang Sun, Yuanyuan Zhang, Fengguang Yang. Collected the date: Mingdong Lu, Shifang Guo. Drafting the manuscript: Wenhui Zhang, Weiqiang Sun.

Acknowledgements: Thanks to gansu Province People's Hospital orthopedics all staff support 


\section{References}

[1] Organization WH. Global Tuberculosis Control: WHO Report 2011. Australian \& New Zealand Journal of Public Health, 2012,36(5): 497-498.

[2] Dara M, Kremer K, Huitric E, et al. Tuberculosis surveillance and monitoring in Europe 2012. Stockholm: European Centre for Disease Prevention and Control, 2012.

[3] Tuli SM. Historical aspects of Pott's disease (spinal tuberculosis) management[J]. Eur Spine J, 2013, 22 Suppl 4: 529-538.

[4] Tuli SM. Tuberculosis of the spine: a historical review[J]. Clin Orthop Relat Res, 2007, 460: 29-38.

[5] Shi T, Zhang Z, Dai F, et al. Retrospective study of 967 patients with spinal Tuberculosis[J]. Orthopedics,2016,39(5): e838-843.

[6] Nene A, Bhojraj S. Results of treatment of thoracic spinal tuberculosis in adult[J].

Spine J, 2005, 5(1): 79-84.

[7] Wang X, Pang X, Wu P, et al. One-stage anterior debridement,bone grafting and posterior instrumentation vs. single posterior debridement, bone grafting, and instrumentation for the treatment of thoracic and lumbar spinal tuberculosis[J]. Eur Spine J,2014,23(4):830-837.

[8] Xu Z, Wang X, Shen X, et al. Posterior only versus combined posterior and anterior approaches for lower lumbar tuberculous spondylitis with neurological deficits in the aged[J]. Spinal Cord,2015,53(6):482-487.

[9] SINGH K, VACCARO A R. Treatment of lumbar instability: transforaminal lumbar interbody fusion[J]. Seminars Spine Surg $₫ 2005 \rrbracket 17$ ( 4) : 259-266】 [10]Seng C『SIDDIGUI M A囚WONG K P\et al『Five-year outcomes of minimally invasive versus open transforaminal lumbar interbody fusion: a matched pair

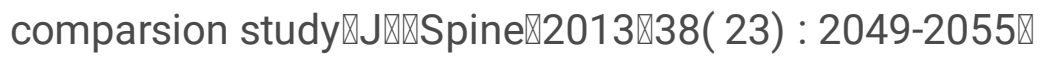

[11] Liu P囚Sun M囚Li S『et al. A retrospective controlled study of three different

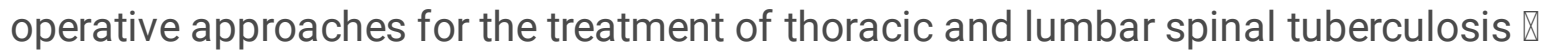


three years of follow-up[J]. Clin Neurol Neurosurg,2015,128:25-34.

[12] Jin W, Wang Q, Wang Z, et al. Complete debridement for treatment of

thoracolumbar spinal tuberculosis: a clinical curative effect observation[J]. Spine J, 2014, 14(6): 964-970.

[13] Yang P, Zang Q, Kang J, et al. Comparison of clinical efficacy and safety among three surgical approaches for the treatment of spinal tuberculosis: a meta-analysis[J].

Eur Spine J, 2016, 25(12): 3862-3874.

[14] ABBASI H $\triangle A B B A S I$ A囚Oblique lateral lumbar interbody fusion ( OLIF ) :

technical notes and early results of a single surgeon comparative study[J]. Cureus $, 2015,7(10):$ e351区

[15] Mihir B, Vinod L, Umesh M, et al. Anterior instrumentation of the cervicothoracic vertebrae: approach based on clinical and radiologic criteria[J]. Spine (Phila Pa 1976), 2006, 31(9): E244-249.

[16] Panchmatia JR, Lenke LG, Molloy S, et al. Review article: Surgical approaches for correction of post-tubercular kyphosis[J]. J Orthop Surg (Hong Kong), 2015, 23(3): 391-394.

[17] Singh K, Vaccaro AR, Kim J, et al. Biomechanical comparison of cervical spine reconstructive techniques after a multilevel corpectomy of the cervical spine[J]. Spine (Phila Pa 1976), 2003, 28(20): 2352-2358.

[18] Azam MQ, Sadat-Ali M. The Concept of Evolution of Thoracolumbar Fracture Classifications Helps in Surgical Decisions[J]. Asian Spine J, 2015, 9(6): 984-994. [19] Liu Z囚Liu J『Peng A『et al. One-stage posterior debridement and transpedicular screw fixation for treating monosegmental thoracic and lumbar spinal tuberculosis in adults[J]. Scientific World J,2014, 13(7):01-06. 
[20] Hassan K, Elmorshidy E. Anterior versus posterior approach in surgical

treatment of tuberculous spondylodiscitis of thoracic and lumbar spine [J]. Eur Spine J, 2016, 25 (4) :1056-1063.

[21] Wang LJ, Zhang HQ, Tang MX, et al. Comparison of three surgical approaches for thoracic spinal tuberculosis in adult \minimum 5-year follow up[J].Spine囚Phila Pa 1976ه,2017,42(11):808-817.

[22] Jin W, Wang Z. Clinical evaluation of the stability of single segment short pedicle screw fixation for the reconstruction of lumbar and sacral tuberculosis lesions[J]. Arch Orthop Trauma Surg,2012, 132(10): 1429-1435.

[23] Zhang HQ, Li JS, Zhao SS, et al. Surgical management for thoracic spinal tuberculosis in the elderly: posterior only versus combined posterior and anterior approaches[J]. Arch Orthop Trauma Surg,2012, 132(12): 1717-1723.

[24] Zeng H, Shen X, Luo C, et al. Comparison of three surgical approaches for cervicothoracic spinal tuberculosis: a retrospective case-control study[J]. J Orthop Surg Res, 2015, 10: 100.

[25] Zhang XF, Wang Y, lao SH, et al. Treatment of lumbar and lumbosacral spinal tuberculosis with minimally invasive surgery[J]. Orthop Surg, 2010, 2(1): 64-70. [26] Li T, Liu T, Jiang Z, et al. Diagnosing pyogenic, brucella and tuberculous spondylitis using histopathology and MRI: a retrospective study[J]. Exp Ther Med, 2016, 12(4): 2069-2077.

[27] Hangli Wu, Yaqing Cui, Liqun Gun, et al. Comparison between single anterior and single posterior approaches of debridement interbody fusion and fxation for the treatment of mono-segment lumbar spine tuberculosis[J]. Archives of Orthopaedic and Trauma Surgery, https://doi.org/10.1007/s00402-021-03955-4. 
[28] Yongchun Zhou, Weiwei Li, Jun Li, et al. Comparison of single posterior

debridement, bone grafting and instrumentation with single-stage anterior

debridement, bone grafting and posterior instrumentation in the treatment of thoracic

and thoracolumbar spinal tuberculosis[J]. MBC Surgery,2018,18:71.

Figures 

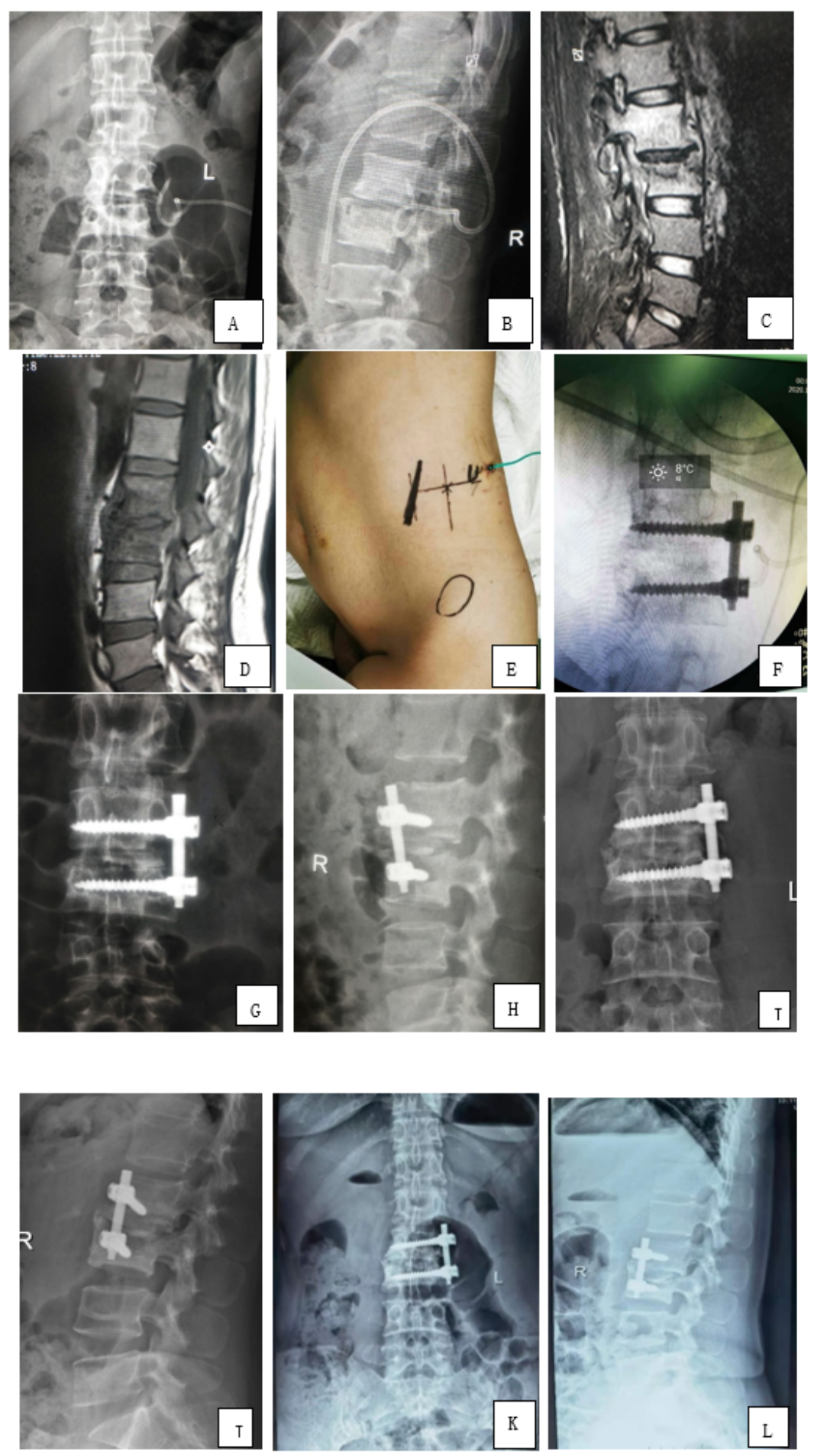

\section{Figure 1}

A 35-year-old woman with lumbar tuberculosis underwent oblique lateral interbody fusion. A, B: Preoperative anteroposterior and lateral radiographs of the lumbar spine showed lesion destruction of the L3 vertebra and narrowing of the L2/3 intervertebral space. A psoas major drainage tube was placed before the surgery. C, D: Preoperative lumbar MRI revealed destruction of L2 and L3 vertebrae, paravertebral abscess formation, and spinal canal compression. E: Preoperative planned surgical 
approach showed that the patient was in lateral decubitus position, and the site of surgical incision and iliac crest was marked. F: Intraoperative fluoroscopy revealed that the intraoperative lesions were removed and the vertebral nails were inserted. Fluoroscopy confirmed that the vertebral nails were in a good position. G, H: Postoperative anteroposterior and lateral lumbar radiographs showed that parts of the lesions in L3 vertebrae were removed after surgery, pre-removed iliac bone was inserted between L2/3 vertebrae, and vertebral screws were used to maintain the stability of the spine. I, J: Six months after the operation, the anterolateral radiographs showed good internal fixation position and good fusion of bone graft. K, L: 12 months after the surgery, anteroposterior and lateral radiographs showed good internal fixation and bone graft fusion, but mild vertebral collapse and backward displacement. 